Review Article

\title{
Perioperative Hemodynamic Management and Pharmacotherapeutics of Patients Undergoing Thoracic Endovascular Aortic Repair
}

\author{
Anja Muehle, Isil Uzun, Ziba Jalali, and Ali Khoynezhad \\ Division of Cardiothoracic Surgery, Cedars-Sinai Medical Center, 8700 Beverly Boulevard, Los Angeles, CA 90048, USA \\ Correspondence should be addressed to Ali Khoynezhad; akhoy@cshs.org
}

Received 30 June 2014; Accepted 16 December 2014; Published 30 December 2014

Academic Editor: David Tanne

Copyright (C) 2014 Anja Muehle et al. This is an open access article distributed under the Creative Commons Attribution License, which permits unrestricted use, distribution, and reproduction in any medium, provided the original work is properly cited.

\begin{abstract}
Thoracic endovascular aortic repair (TEVAR) has become an attractive alternative treatment option for many patients with specific thoracic aortic disease. New devices and advanced image-guided procedures are continuously expanding the indications and improve neurological outcomes. Hemodynamic management of these patients is a critical aspect in reducing neurological deficit and it is different compared to patients undergoing open thoracic aortic operations. There are two different phases of blood pressure management for patients with thoracic aortic disease. Before and during the critical steps of TEVAR anti-impulsive therapy facilitates safe positioning and stent deployment. After stent grafts are deployed, controlled hypertensive blood pressure levels are achieved to avoid spinal cord ischemia. This precise blood pressure strategy is essential to ensure a safe procedure and good longterm results.
\end{abstract}

\section{Introduction}

Since 1991, Parodi et al. described the first endovascular exclusion of an abdominal aortic aneurysm [1]; the technique and devices evolved rapidly and thoracic aortic diseases were involved. Dake et al. introduced in 1994 the use of stent grafts for the treatment of the descending thoracic aortic aneurysm for high risk patients [2] and Shimono et al. in 1998 for an acute type A aortic dissection [3]. Since the first stent grafts for thoracic endovascular aortic repair (TEVAR) were approved and become commercially available in 2005, feasibility and successful utilization of this technique have been established. Endovascular repair in selected patients with complex aortic pathologies has now lower perioperative mortality and complications compared with open surgical repair [4-6].

Besides the interventional treatment, in patients with thoracic aortic disease stringent antihypertensive therapy, lipid profile optimization, smoking cessation, and reduction of other atherosclerosis risk factors are recommended [7]. This accompanying medical therapy has evolved and their benefits were studied on the effect of aneurysm growth.
Furthermore, blood pressure management plays a critical role during the TEVAR procedure itself and demands a close collaboration between the surgeon and anesthesiologist as well as an understanding of each specialty and interventional steps.

The aim of this is to provide a concise update on various aspects of thoracic endografting followed by a more comprehensive review of the antihypertensive medications used for hemodynamic management of the patients with aortic disease and during the endovascular procedure.

\section{Preoperative Imaging}

Preoperative planning with different imaging utilities is critical for TEVAR. CT scans or MRI provides detailed information about the aortic anatomy including the size and quality of the aortic wall. Furthermore it allows an assessment of the landing zones, size, and angulation of access vessels and also demonstrates the location of vital side branches.

Intravascular ultrasound (IVUS) is valuable intraoperative tool for TEVAR procedures, especially in aortic dissection [8]. This technology can be used to identify the position 
of branch vessels, inspect vessel wall morphology, evaluate the presence of plaques or thrombi, and select appropriate landing zones for endografts. A particularly important application of IVUS is confirmation of the true vessel lumen in aortic dissection and after deployment of the endograft it confirms the complete apposition of the device to the aortic wall without any endoleaks. IVUS can significantly reduce the amount of contrast material used and fluoroscopy exposure.

Angulation of the arch with a small inner curve and a large outer curve (gothic arch) is another critical issue. The endovascular treatment of these aortic arches with a steep angulation $>90^{\circ}$ with commercially available stent grafts is associated with an increased risk of stent graft collapse, retrograde type A dissection, and type Ia endoleak $[9,10]$. The primary cause of endograft collapse in a small radius of curvature of the aortic arch is excessive oversizing [9]. Dumfarth et al. recommend a careful consideration of TEVAR in these cases and avoiding the use of the angulated segment as a landing zone if possible [10].

Another anatomic challenge is a wide difference between proximal and distal landing zones. This can appear especially in extensive thoracic-abdominal aortic pathologies. In these cases, carefully imaged and properly planned stent graft tapered configurations, or custom-made devices, will address an adequate stent graft alignment over the whole length of the aorta [11].

\section{Endovascular Solutions}

In patients with aortic aneurysms that require fixation or landing zones within the aortic arch, endovascular repair requires either a hybrid two-staged operation with open surgical debranching followed by endovascular graft exclusion or a single stage endovascular repair using either branched or fenestrated stent grafts.

Chimney stent grafting is an endovascular technique of maintaining perfusion to aortic side branches which would require coverage by the aortic stent graft in order to obtain an adequate seal zone. It was initially developed to preserve renal arteries in patients with abdominal aortic aneurysms with short proximal necks by Greenberg and colleagues [12]. One stent is placed in a side branch at the proximal or distal seal zone and another bigger main stent graft into the aorta. Then both stent grafts are kept parallel and deployed simultaneously. One of the most technical concerns of chimney stent grafting is the possibility of a type I endoleak. To prevent the leakage through the gutter between the chimney graft and the main aortic graft appropriate oversizing of the aortic stent graft is critical.

\section{Complications}

TEVAR is associated with a wide spectrum of complications, and our preventive and management strategies have evolved over the years. While vascular injuries remain the most serious complication in patients undergoing abdominal aortic aneurysm, the most serious complications of TEVAR are neurologic that can be due to ischemia or embolization.
Paraplegia or paraparesis may occur due to temporary or permanent spinal cord ischemia (SCI) and perioperative acute ischemic stroke [13]. The risk of permanent spinal cord injury ranges from 0 to $4.5 \%$ [14]. The EUROSTAR group [15] identified independent risk factors for SCI, such as left subclavian artery coverage without revascularization, renal failure, concomitant open abdominal aorta surgery, and the use of three or more stent grafts. Perioperative acute ischemic stroke has an incidence from $3.5 \%$ to $5.5 \%[16,17]$ and is more common if the TEVAR involves more proximal aorta (landing zones 0-2) [18]. Excessive wire and catheter manipulation in a diseased aortic arch with air embolism from deployment systems and inadvertent coverage of arch branches (carotid arteries and the left subclavian artery) increases the risk of stroke. There is more evidence that sufficient perfusion of the vertebral arteries (by prophylactic subclavian revascularization) is the best approach to prevent ischemic events in the brainstem and the posterior cerebral circulation [19].

Another risk after TEVAR is the presence of endoleaks, characterized by the persistence of blood flow within the aneurysm sac. The incidence of endoleaks following TEVAR in a recent meta-analysis of reported studies is $12.1 \%$ [5]. It can be classified into 5 types [20, 21]: type I, leak at the anastomotic site of the aorta and the stent graft; type II, collateral vessels that communicate within the aneurysm sac; type III, stent-graft junction leak; type IV, leak through the stent-graft fabric due to graft-material porosity; and type $\mathrm{V}$ or endotension, persistent pressurization of the aneurysmal sac without evidence of perigraft blood flow. Endoleak types I and III are a result of technical failure and require immediate intervention. Type I has been associated with early mortality in multivariate analysis and has to be corrected intraoperatively, whenever possible [16]. It may be treated with either additional balloon dilatation or an additional stent graft to extend the proximal or distal landing zone. Endoleak type II does not need further intervention in the absence of aneurysm enlargement.

\section{Medical Management}

Initial management of patients with thoracic aortic disease involves safe and strict control of hypertension to reduce shear forces on the aortic wall and to prevent or slow further progression of the aneurysm or dissection and possible rupture $[7,22]$.

Many patients with thoracic aortic disease are at higher risk for aortic rupture presenting hypertensive crisis and/or emergency, requiring prompt and aggressive therapy to control the blood pressure and further organ damage. Hypertensive crisis has been defined as a systolic blood pressure > $180 \mathrm{mmHg}$ or a diastolic blood pressure $>120 \mathrm{mmHg}$ [23]. Presence or absence of end-organ damage classifies it as emergency or urgency. Organ damage can be neurological such as hypertensive encephalopathy, stroke, or intracranial hemorrhage, but it can also lead to acute heart failure, pulmonary edema, aortic dissection, renal failure, or eclampsia. Because of its bad prognosis in the past of about $80 \%$ 
mortality at 1 year, hypertensive crisis was initially named malignant hypertension. With advances in medical therapy and adequate control of the blood pressure mortality rates decrease to $10 \%$ at 1 year.

Various drugs with different action sites and effects have been used: vasodilators, calcium channel blockers, angiotensin converting enzyme inhibitors or angiotensin receptor blockers, and $\beta$-blockers.

The agent of choice and the rate of decreasing the blood pressure depend on the clinical presentation and the mainly affected organ system. Blood pressure in acute stroke should be lowered extremely cautiously, but aggressively in acute pulmonary edema due to hypertensive heart failure or aortic regurgitation secondary to type A aortic dissection. For patients with aortic disease $\beta$-blockers are preferred before vasodilators use because they decrease heart rate and left ventricular contractility as desired and do not cause reflex tachycardia due to vasodilatation.

In endovascular repair meticulous blood pressure control is crucial to allow a safe positioning of the stent, without migration during deployment or damage to the aortic wall. During the short period of aortic occlusion and stent-graft deployment, the patient should be kept hypotensive and bradycardic, if possible. But after the stent graft is inserted the blood pressure has to be maintained on the higher level to secure a good spinal cord perfusion and avoid any ischemia. For this challenging hemodynamic management, intravenous short acting antihypertensive management is preferred.

5.1. Vasodilators. Nitroprusside works through release of nitric oxide and has a rapid onset of action within seconds to minutes. Because of its simultaneous arterial and venodilatation the hypotensive effects can be unpredictable, especially in patients with severe ventricular hypertrophy and preload-dependent diastolic dysfunction [24]. Furthermore, it can cause coronary steal or reflex tachycardia. But the most serious adverse effect is the cyanide toxicity, which occurs due to accumulation of its metabolite cyanide. Therefore it should be given only as a last resource and accompanied with sodium thiosulfate that acts as the sulfate donor to convert cyanide in thiocyanate.

Nitroglycerin releases nitric oxide and causes vasodilatation mainly in the venous system. Its onset of action is within minutes, but patients develop tolerance to it and it is not effective for prolonged use.

5.2. Calcium Channel Blocker. Data from the IRAD showed that the use of calcium channel blockers was associated with an improved survival in patients with aortic dissection type B [25].

Nicardipine is the only FDA-approved short-acting calcium channel blocker belonging to the dihydropyridine class. It causes cerebral and coronary vasodilatation with only minimal negative inotropic and chronotropic effects. In contrast to nitroprusside, the pharmacodynamics properties of nicardipine are favorable, but their pharmacokinetic properties are not. Nicardipine has a very long half-life; its $\beta$-half-life is $40 \mathrm{~min}$, but its $\gamma$-half-life is approximately $13 \mathrm{~h}$ and $14 \%$ of the drug is eliminated during the $\gamma$-phase [24]. Therefore the hypotensive effect can be prolonged. In a study comparing the effect of nicardipine to nitroprusside in patients with severe postoperative hypertension, the two drugs had equivalent efficiency, but only nicardipine reduced both cardiac and cerebral ischemia [26].

5.3. Dopamine-1 Agonist. Fenoldopam is a selective dopamine-1 agonist and selective arteriolar/renal dilator. It has a rapid onset of action and a short duration of action, with both about 5 minutes. Because it improves creatinine clearance, urine flow rates, and sodium excretion in severely hypotensive patients with impaired renal function, fenoldopam may be the drug of choice in these patients [27]. Compared to nitroprusside, fenoldopam has a longer half-life and does not cause venodilatation; thus blood pressure reduction is more predictable. But like other vasodilators, fenoldopam may cause reflex tachycardia and nonspecific T-wave and ST changes on the ECG. It may reduce serum potassium and produces a mild tolerance after long-term infusion [24, 28].

5.4. $\beta$-Blocker. Beta adrenergic-blocking drugs are recommended in most patients with aortic pathologies because they are not only an effective anti-impulsive medication, but they also reduce myocardial oxygen consumption, thereby reducing coronary ischemia.

In aortic dissection and aortic aneurysm, propagation of aortic dissection depends not only on the absolute blood pressure, but also on the velocity of the left ventricular contraction [29]. Instead of decreasing the heart rate, a vasodilator alone may even cause reflex tachycardia, thus causing propagation of the dissection. Therefore, the optimum treatment involves a combination of a parenteral $\beta$-blocker and a vasodilator, with heart rate targeted at 60 beats/min [28]. The $\beta$-blocker of choice in this situation is generally esmolol and, alternatively, labetalol or metoprolol [29].

Esmolol is a $\beta 1$-receptor antagonist while labetalol is a combined $\alpha 1-, \beta 1-$, and $\beta 2$-receptor antagonist with an alpha to beta blocking ratio of $1: 7$ [30]. Both of them, by slowing down the heart rate, also reduce the myocardial oxygen demand. Esmolol reduces blood pressure by reducing cardiac output and inhibiting renin release, while labetalol decreases afterload directly and also inhibits renin release [24]. Disadvantages of these drugs are their negative inotropic effect and possible reaction in patients with reactive airway disease. Both $\beta$-blockers have a rapid onset of action, but esmolol has a much shorter half-life of 9 minutes compared to 5.5 hours of labetalol.

A combination of a short-acting intravenous $\beta$-blocker and a vasodilator is the preferred initial approach for many patients. Thereby, $\beta$-blockers should be given first before vasodilators, as vasodilatation can lead to reflex release of catecholamines and therefore to tachycardia and increase ventricular contractions. Addition of calcium antagonist and a second parenteral beta blocker are next steps in management of patients with resistant hypertension. 


\section{Periprocedural Hemodynamic Management}

Preoperatively, clonidine or beta blockers can and should be continued due to potential rebound of blood pressure or heart rate due to sudden withdrawal [23]. Because of its potential adverse interaction with surgery-dependent fluid depletion, diuretics should be avoided on the day of surgery. Furthermore, ACE inhibitors and angiotensin receptor blockers should be stopped two days prior to elective TEVAR procedures, as they can precipitate intraoperative vasoplegia and hard-to-treat hypotension [31].

During the TEVAR procedure itself, immediately before critical steps such as aortic occlusion and stent-graft deployment, cardiac output should be minimized to lower backflow and movements of the device and promote the accuracy of deployment. This can be achieved by administration of adenosine that causes a transient heart block in preparation for stent deployment in the mid and proximal aortic arch. The usual dose is $36 \mathrm{mg}$ of adenosine (followed by $18 \mathrm{mg}$ for subsequent episodes) that will produce a cardiac asystole for a period of 4-6 seconds [32]. This transient asystole reduces the $\mathrm{dp} / \mathrm{dt}$ in the aorta and the "windsocking" reducing the chance of malposition and inadvertent coverage of vital brachiocephalic arteries.

Rapid pacing is another method to lower the cardiac excursion during the balloon inflation and stent deployment. In these cases, a temporary transvenous pacing electrode will be placed via the jugular vein before the procedure and then for rapid pacing the heart is stimulated with 160180 beats/min. Upon deployment of the stent graft, the rapid pacing is stopped, and the blood pressure is recovered.

Both adenosine and rapid right-ventricular pacing have the benefit of being very short-acting. Upon stent-graft deployment, changing patient's hemodynamics is more expeditious compared to using short-acting beta blocker or calcium antagonist [22].

At this portion of the TEVAR procedure, the blood pressure is increased, the cardiac output is increased, and (on occasion) the hemoglobin concentration is increased to improve blood oxygen delivery to spinal cord. Typically, the patient is allowed briefly to recover from the antihypertensive treatment modalities, aiming for mean blood pressure in $80 \mathrm{~s}$ to $90 \mathrm{~s} \mathrm{mmHg}$ to reduce the spinal cord ischemia [33]. Increased blood pressure improves the spinal cord perfusion pressure in the anterior spinal artery across the stent-grafted segment of the aorta that now has lack of perfusion from the intercostal arteries $[4,13]$. If the desired blood pressure is not achieved or there is evidence of spinal cord ischemia of the motor or sensory evoked potentials, the blood pressure is increased using vasopressin or catecholamines. Compared to catecholamines, vasopressin increases the systemic blood pressure without increasing the heart rate. This is of obvious advantage in patients with coronary artery disease that compromise the majority of patients undergoing TEVAR.

If, despite increasing blood pressure and cardiac output, there is concern or neuromonitoring evidence of ongoing spinal cord injury, we drain cerebrospinal fluid from patient's spinal cord drainage catheter to reduce spinal fluid pressure. Since the resulting perfusion pressure is the difference between the blood pressure and the intracranial pressure (ICP), decreasing the ICP between 10 and $12 \mathrm{mmHg}$ will improve the perfusion. The ICP can be controlled by monitoring and active drainage either with an automatic continuous draining machine or manually every hour. Interestingly, a recent retrospective study by Jafarzadeh et al. showed that intravenously given acetazolamide can reduce cerebrospinal fluid (CSF) production in patients with thoracoabdominal aortic surgery [34] too. In this study the administration of acetazolamide not only decreases the CSF production and the ICP but also improved the paraplegia.

These interventions are all aimed at improving spinal cord perfusion pressure [35]. This hypertensive management is typically continued for the first 48 hours after the endografting, and the patient is kept slightly more hypertensive for another two weeks, before more strict blood pressure control is persuaded. This is in opposite to postoperative management of patients undergoing open aortic operations, where the blood pressure is kept strictly on the lower side to reduce anastomotic bleeding complications [28].

In addition to the pressure and perfusion management, the oxygen supply to the endangered spinal cord can be improved by increasing the oxygen-transport capacity by transfusion of erythrocytes if the hemoglobin is below $10 \mathrm{mg} / \mathrm{dL}$.

Aforementioned, targeted pharmacotherapeutics and individualized hemodynamic management by experienced anesthesiologists and intensivists were important steps in reducing adverse events and neurological complications in patients undergoing TEVAR.

\section{Conclusion}

Endovascular repair for complex thoracic aortic pathologies has evolved in the last years with continuously expanding indications and improved neurological outcomes. Optimal and individualized hemodynamic management is essential for improved outcomes. Precise control of the blood pressure during the various stages of the intervention will facilitate stent-graft delivery, improve accuracy of deployment, and reduce neurological complications.

\section{Conflict of Interests}

The authors declare that there is no conflict of interests regarding the publication of this paper.

\section{References}

[1] J. C. Parodi, J. C. Palmaz, and H. D. Barone, "Transfemoral intraluminal graft implantation for abdominal aortic aneurysms," Annals of Vascular Surgery, vol. 5, no. 6, pp. 491-499, 1991.

[2] M. D. Dake, D. C. Miller, C. P. Semba, R. S. Mitchell, P. J. Walker, and R. P. Liddell, "Transluminal placement of endovascular stent-grafts for the treatment of descending thoracic aortic aneurysms," The New England Journal of Medicine, vol. 331, no. 26, pp. 1729-1734, 1994.

[3] T. Shimono, N. Kato, T. Tokui et al., "Endovascular stent-graft repair for acute type A aortic dissection with an intimal tear in 
the descending aorta," Journal of Thoracic and Cardiovascular Surgery, vol. 116, no. 1, pp. 171-173, 1998.

[4] A. Khoynezhad, C. E. Donayre, J. Smith, G. E. Kopchok, I. Walot, and R. A. White, "Risk factors for early and late mortality after thoracic endovascular aortic repair," Journal of Thoracic and Cardiovascular Surgery, vol. 135, no. 5, pp. 1103.e1-1109.e4, 2008.

[5] D. Cheng, J. Martin, H. Shennib et al., "Endovascular aortic repair versus open surgical repair for descending thoracic aortic disease a systematic review and meta-analysis of comparative studies," Journal of the American College of Cardiology, vol. 55, no. 10, pp. 986-1001, 2010.

[6] A. Khoynezhad, C. E. Donayre, B. O. Omari, G. E. Kopchok, I. Walot, and R. A. White, "Midterm results of endovascular treatment of complicated acute type B aortic dissection," Journal of Thoracic and Cardiovascular Surgery, vol. 138, no. 3, pp. 625631, 2009.

[7] L. F. Hiratzka, G. L. Bakris, J. A. Beckman et al., "2010 ACCF/ AHA/AATS/ACR/ASA/SCA/SCAI/SIR/STS/SVM guidelines for the diagnosis and management of patients with Thoracic Aortic Disease: a report of the American College of Cardiology Foundation/American Heart Association Task Force on Practice Guidelines, American Association for Thoracic Surgery, American College of Radiology, American Stroke Association, Society of Cardiovascular Anesthesiologists, Society for Cardiovascular Angiography and Interventions, Society of Interventional Radiology, Society of Thoracic Surgeons, and Society for Vascular Medicine," Circulation, vol. 121, no. 13, pp. e266-e369, 2010.

[8] A. Khoynezhad, R. Celis, M. Hashemzadeh, M. R. Movahed, and Z. Jalali, "Current status of thoracic endografting and its adjunctive pharmacology," Expert Opinion on Pharmacotherapy, vol. 10, no. 17, pp. 2871-2881, 2009.

[9] F. H. W. Jonker, F. J. V. Schlosser, A. Geirsson, B. E. Sumpio, F. L. Moll, and B. E. Muhs, "Endograft collapse after thoracic endovascular aortic repair," Journal of Endovascular Therapy, vol. 17, no. 6, pp. 725-734, 2010.

[10] J. Dumfarth, M. Michel, J. Schmidli et al., "Mechanisms of failure and outcome of secondary surgical interventions after thoracic endovascular aortic repair (TEVAR)," The Annals of Thoracic Surgery, vol. 91, no. 4, pp. 1141-1146, 2011.

[11] P. de Rango, "Total endovascular repair for thoraco-abdominal aneurysms: not for all, not for now," European Journal of Vascular and Endovascular Surgery, vol. 43, no. 3, p. 268, 2012.

[12] R. K. Greenberg, S. Haulon, S. O’Neill, S. Lyden, and K. Ouriel, "Primary endovascular repair of juxtarenal aneurysms with fenestrated endovascular grafting," European Journal of Vascular and Endovascular Surgery, vol. 27, no. 5, pp. 484-491, 2004.

[13] A. Khoynezhad, P. Upadhyaya, and M. J. Kruse, "Spinal cord injury and stroke following thoracic endovascular aortic repair: a risk analysis and review of the literature," Italian Journal of Vascular and Endovascular Surgery, vol. 14, no. 3, pp. 221-229, 2007.

[14] W. A. Lee, J. S. Matsumura, R. S. Mitchell et al., "Endovascular repair of traumatic thoracic aortic injury: clinical practice guidelines of the society for vascular surgery," Journal of Vascular Surgery, vol. 53, no. 1, pp. 187-192, 2011.

[15] J. Buth, P. L. Harris, R. Hobo et al., "Neurologic complications associated with endovascular repair of thoracic aortic pathology: incidence and risk factors. A study from the European Collaborators on Stent/Graft Techniques for Aortic Aneurysm
Repair (EUROSTAR) Registry," Journal of Vascular Surgery, vol. 46, no. 6, pp. 1103.e2-1111.e2, 2007.

[16] A. Khoynezhad, C. E. Donayre, H. Bui, G. E. Kopchok, I. Walot, and R. A. White, "Risk factors of neurologic deficit after thoracic aortic endografting," Annals of Thoracic Surgery, vol. 83, no. 2, pp. S882-S889, 2007.

[17] B. A. Ehlert, C. A. Durham, F. M. Parker, W. M. Bogey, C. S. Powell, and M. C. Stoner, "Impact of operative indication and surgical complexity on outcomes after thoracic endovascular aortic repair at National Surgical Quality Improvement Program Centers," Journal of Vascular Surgery, vol. 54, no. 6, pp. 1629.e1-1636.el, 2011.

[18] G. Melissano, A. Kahlberg, L. Bertoglio, and R. Chiesa, "Endovascular exclusion of thoracic aortic aneurysms with the 1- and 2-component Zenith TX2 TAA endovascular grafts: analysis of 2-year data from the TX2 pivotal trial," Journal of Endovascular Therapy, vol. 18, no. 3, pp. 338-349, 2011.

[19] L. Canaud, K. Hireche, J.-P. Berthet, P. Branchereau, C. MartyAné, and P. Alric, "Endovascular repair of aortic arch lesions in high-risk patients or after previous aortic surgery: midterm results," Journal of Thoracic and Cardiovascular Surgery, vol. 140, no. 1, pp. 52-58, 2010.

[20] S. W. Stavropoulos and J. P. Carpenter, "Postoperative imaging surveillance and endoleak management after endovascular repair of thoracic aortic aneurysms," Journal of Vascular Surgery, vol. 43, supplement, no. 2, pp. A89-A93, 2006.

[21] M. I. Turina, H. Shennib, J. Dunning et al., "EACTS/ESCVS best practice guidelines for reporting treatment results in the thoracic aorta," European Journal of Cardio-thoracic Surgery, vol. 35, no. 6, pp. 927-930, 2009.

[22] P. K. Gupta, H. Gupta, and A. Khoynezhad, "Hypertensive emergency in aortic dissection and thoracic aortic aneurysm-a review of management," Pharmaceuticals, vol. 2, no. 3, pp. 6676, 2009.

[23] G. Mancia, R. Fagard, K. Narkiewicz et al., "2013 ESH/ESC guidelines for the management of arterial hypertension: the Task Force for the Management of Arterial Hypertension of the European Society of Hypertension (ESH) and of the European Society of Cardiology (ESC)," European Heart Journal, vol. 34, pp. 2159-2219, 2013.

[24] S. Oparil, "Treating multiple-risk hypertensive populations," The American Journal of Hypertension, vol. 12, no. 11, pp. 121S129S, 1999.

[25] T. Suzuki, E. M. Isselbacher, C. A. Nienaber et al., "Typeselective benefits of medications in treatment of acute aortic dissection (from the International Registry of Acute Aortic Dissection [IRAD])," The American Journal of Cardiology, vol. 109, no. 1, pp. 122-127, 2012.

[26] N. A. Halpern, R. N. Sladen, J. S. Goldberg et al., "Nicardipine infusion for postoperative hypertension after surgery of the head and neck," Critical Care Medicine, vol. 18, no. 9, pp. 950955, 1990.

[27] N. H. Shusterman, W. J. Elliott, and W. B. White, "Fenoldopam, but not nitroprusside, improves renal function in severely hypertensive patients with impaired renal function," The American Journal of Medicine, vol. 95, no. 2, pp. 161-168, 1993.

[28] A. Khoynezhad and K. A. Plestis, "Managing emergency hypertension in aortic dissection and aortic aneurysm surgery," Journal of Cardiac Surgery, vol. 21, supplement 1, pp. S3-S7, 2006.

[29] J. Varon and P. E. Marik, "The diagnosis and management of hypertensive crises," Chest, vol. 118, no. 1, pp. 214-227, 2000. 
[30] P. Lund-Johansen and P. Omvik, "Prizidilol in essential hypertension: long-term effects on plasma volume, extracellular fluid volume, and central hemodynamics at rest and during exercise," Journal of Cardiovascular Pharmacology, vol. 4, no. 6, pp. 10121017, 1982.

[31] S. G. Raja and N. Fida, "Should angiotensin converting enzyme inhibitors/angiotensin II receptor antagonists be omitted before cardiac surgery to avoid postoperative vasodilation?" Interactive Cardiovascular and Thoracic Surgery, vol. 7, no. 3, pp. 470-475, 2008.

[32] M. Lippmann, C. Kakazu, T. D. Fang, H. Bui, C. Donayre, and R. A. White, "Adenosine's usefulness in vascular surgery," Texas Heart Institute Journal, vol. 34, no. 2, pp. 258-259, 2007.

[33] A. Khoynezhad, R. Bello, D. R. Smego, L. Nwakanma, and K. A. Plestis, "Improved outcome after repair of descending and thoracoabdominal aortic aneurysms using modern adjuncts," Interactive Cardiovascular and Thoracic Surgery, vol. 4, no. 6, pp. 574-576, 2005.

[34] F. Jafarzadeh, M. L. Field, D. K. Harrington et al., "Novel application of acetazolamide to reduce cerebrospinal fluid production in patients undergoing thoracoabdominal aortic surgery," Interactive Cardiovascular and Thoracic Surgery, vol. 18, no. 1, pp. 21-26, 2014.

[35] H. J. Safi, M. P. Campbell, C. C. Miller III et al., "Cerebral spinal fluid drainage and distal aortic perfusion decrease the incidence of neurological deficit: the results of 343 descending and thoracoabdominal aortic aneurysm repairs," European Journal of Vascular and Endovascular Surgery, vol. 14, no. 2, pp. 118-124, 1997. 


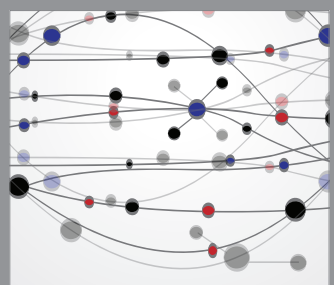

The Scientific World Journal
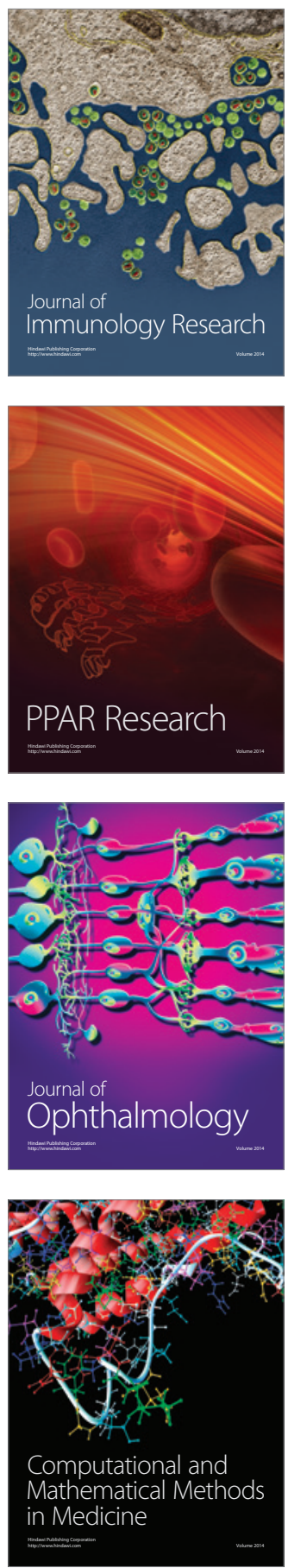

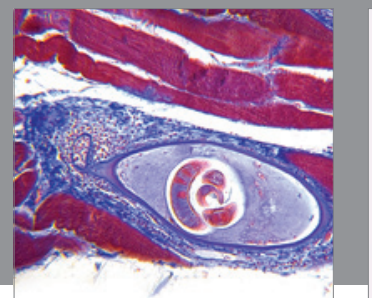

Gastroenterology

Research and Practice
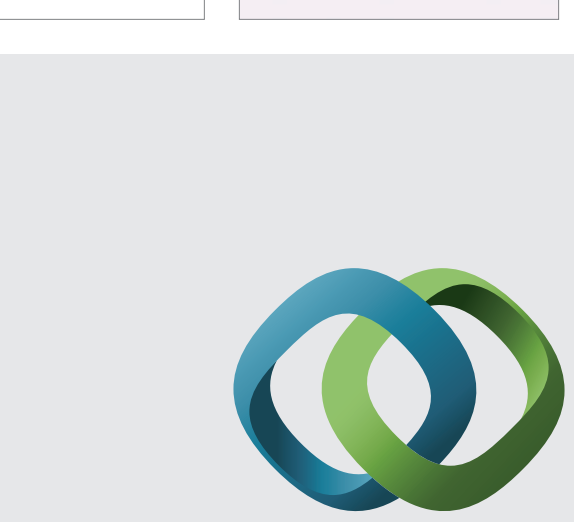

\section{Hindawi}

Submit your manuscripts at

http://www.hindawi.com
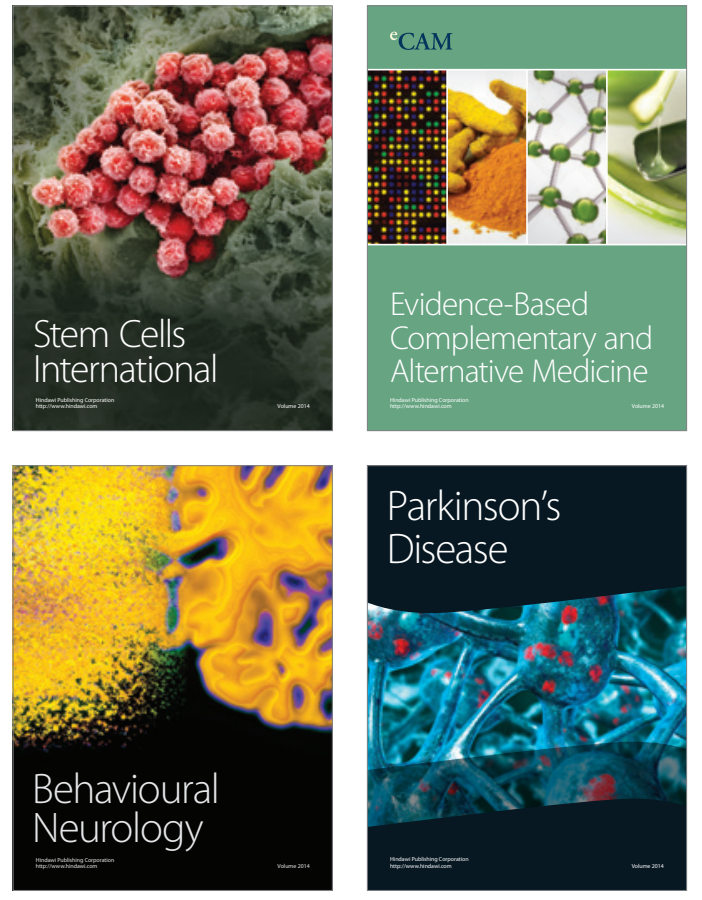
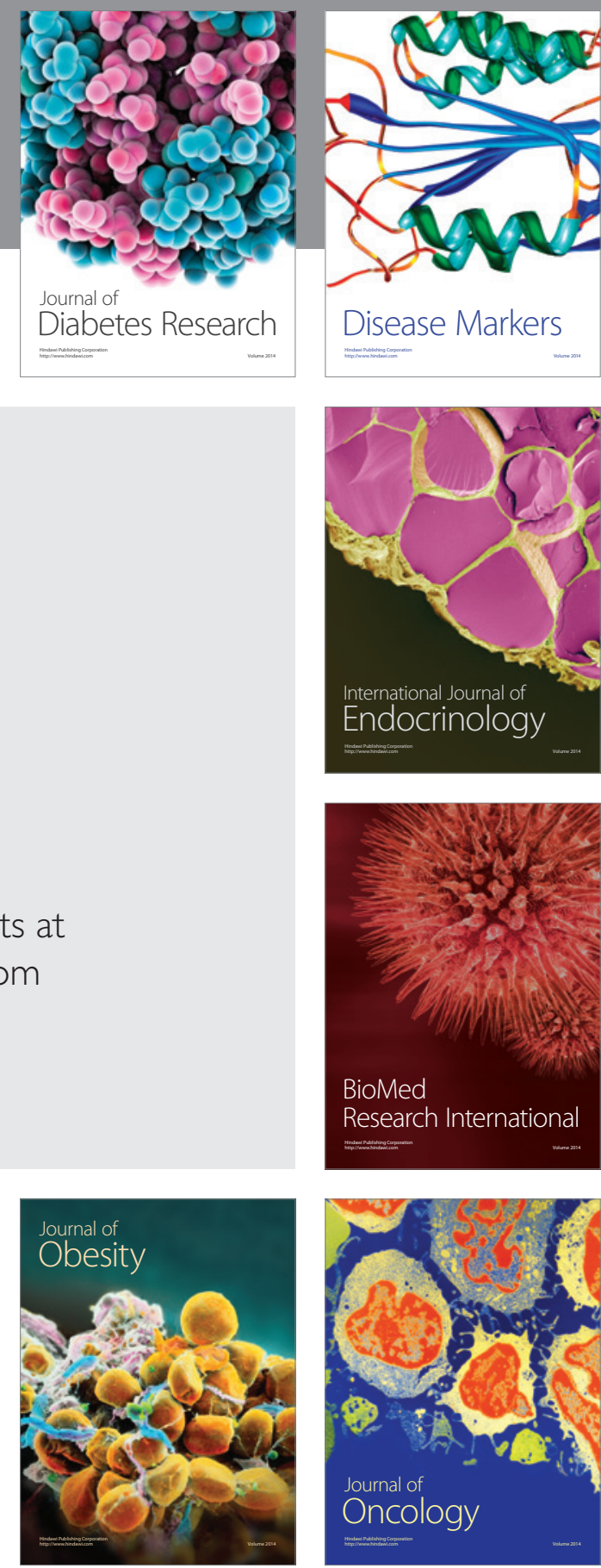

Disease Markers
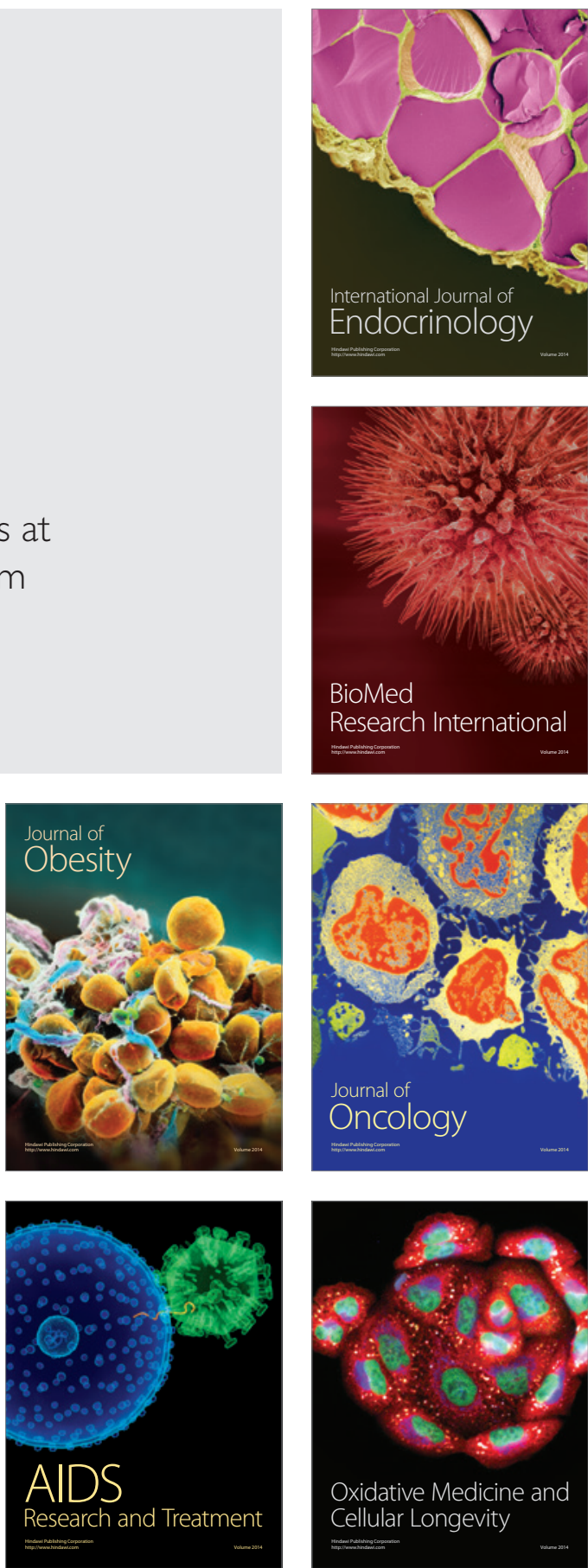